\title{
Are we addressing the top 10 research priorities in IBD?
}

\author{
Jeroen Geldof (D) , ${ }^{1}$ Jean-Frédéric LeBlanc, ${ }^{1}$ Laura Lucaciu, ${ }^{2}$ \\ Jonathan Segal (D) ,' Charlie W Lees, ${ }^{2,3}$ Ailsa Hart ${ }^{1}$
}

\begin{abstract}
${ }^{1}$ IBD unit, St Mark's Hospital and Academic Institute, Harrow, UK ${ }^{2}$ IBD-unit, Western General Hospital, Edinburgh, UK ${ }^{3}$ Centre for Genomics and Experimental Medicine, The University of Edinburgh MRC Institute of Genetics and Molecular Medicine, Edinburgh, UK
\end{abstract}

\section{Correspondence to} Dr Jeroen Geldof, IBD unit, St Mark's Hospital and Academic Institute, Harrow, London, UK; jeroen.geldof@ugent.be

Received 4 June 2020 Revised 28 October 2020 Accepted 29 October 2020

\section{Check for updates}

(c) Author(s) (or their employer(s)) 2020. No commercial re-use. See rights and permissions. Published by BMJ.

To cite: Geldof J, LeBlanc JF, Lucaciu L, et al. Frontline Gastroenterology Epub ahead of print: [please include Day Month Year]. doi:10.1136/ flgastro-2020-101579

\section{ABSTRACT}

Background Since publication of the top 10 research priorities in inflammatory bowel disease (IBD) based on the James Lind Alliance Priority Setting Partnership, the question remains whether this has influenced the IBD-research landscape. This study aimed to create an overview of the current distribution of research interests of trials in the UK.

Methods The ClinicalTrials.gov database and European Union Clinical Trials Register were screened for clinical trials set up from 9 August 2016 to 16 November 2019 in the UK involving adult patients with IBD.

Results Of 20 non-industry-sponsored studies, a quarter investigated treatment strategies considering efficacy, safety and costeffectiveness (priority 1). Four evaluated the role of diet (priorities 3 and 7). Development/ assessment of biomarkers for patient stratification (priority 2) and fatigue (priority 8) were subject of three studies. IBD-related pain and control of diarrhoea/incontinence were each subject of 2 studies (priorities 4 and 6). The effect of gut microbiota (priority 10) and optimal strategy for perianal Crohn's disease (priority 5) was the focus of 2 studies each. One study evaluated surgery for terminal ileal Crohn's disease (priority 9). Of 63 industry-sponsored studies, 59 focused on priority 1 .

Conclusions This study presents an impression of the breadth of the IBD-research landscape in the UK, in light of the top 10 research priorities published in 2016. Optimal treatment strategy has been the most studied research priority by academic and industry-sponsored trials. Fewer studies focused on patient-reported outcomes. It remains debatable to what extent the current research landscape adequately represents all stakeholders' viewpoints on needs for expanded knowledge in $\mathrm{IBD}$, particularly the patients' perspective.

\section{BACKGROUND}

Inflammatory bowel diseases (IBDs) are chronic relapsing and remitting conditions characterised by inflammation of

\section{Key messages}

What is already known on this topic

- In 2016, the top 10 research priorities in inflammatory bowel disease (IBD) were published based on the James Lind Alliance Priority Setting Partnership in the UK.

\section{What this study adds}

- This study shows over the past 3 years that multiple clinical trials have been set up in the UK addressing the top 10 research priorities in IBD, mainly focusing on development and assessment of therapeutic strategies (priority 1). Other research priorities, and especially patient-reported outcomes such as IBDrelated pain, fatigue and management of diarrhoea/incontinence are also addressed, but less frequently.

\section{How might it impact on clinical} practice in the foreseeable future

- It remains debatable to what extent the current research landscape adequately represents all stakeholders' viewpoints on needs for expanded knowledge in IBD, particularly the patients' perspective.

the gastrointestinal tract. Ulcerative colitis (UC) was first described in 1859 by Sir Samuel Wilks. In 1913, a case series of interstitial enteritis was published in the British Medical Journal (BMJ) by Scottish surgeon Dalziel. ${ }^{1}$ It took until 1932, when Burrill B. Crohn, Leon Ginzburg and Gordon D. Oppenheimer published their landmark article, to identify what became known as Crohn's disease. ${ }^{2}$

Since publication of these historical documents, numerous research efforts have been made to clarify the optimal management of these diseases. ${ }^{3}{ }^{4}$ Sulfasalazine, a combination of 5-ASA and sulfapyridine, was synthesised by the Swedish physician Nana Svartz in 1940 
and first used to treat people with arthritis and subsequently those with UC. ${ }^{5}$ Corticosteroids were shown to improve symptoms and reduce mortality in UC in a randomised controlled trial by Truelove and Witts. ${ }^{6}$ Immunosuppressants were first shown to be effective in IBD in the 1960s using thiopurines and in 1980s with methotrexate. ${ }^{7}$ Surgery has been used throughout; the original descriptions were all of resection specimens. Techniques have been refined over decades, with techniques such as ileostomy and subtotal or total colectomy being standardised in 1930s. A better understanding of immunology, molecular biology and genetics of IBD has led to the development of the first biological therapies based on anti-tumour necrosis factor (anti-TNF) inhibition, such as infliximab and adalimumab. ${ }^{8}$ The successful use of these therapies has in part been due to a shift in the focus of treatment from only symptom control to a 'treat to target' approach: including normalisation of biomarkers, mucosal healing, histological healing and healing on abdominal imaging. ${ }^{9}$ Furthermore, standards of IBD care have risen with the implementation of an IBD multidisciplinary team involving GI consultants, surgeons, specialist nurses, dietitians, psychologists, pathologists and radiologists. ${ }^{10}$

Nonetheless, many unanswered questions remain in a variety of aspects of IBD to include prevalence, aetiology, optimal treatments and treatment targets as well as priorities from a patients perspective. ${ }^{11-14}$ In order to help prioritise research into these areas, as of 2014 , a group of multidisciplinary clinicians, patients and patient-support organisations based in the UK collaborated in order to outline the top 10 research priorities in the treatment of IBD using the infrastructure of the James Lind Alliance (JLA) Priority Setting Partnership (PSP).

The JLA PSP provides a framework based on a transparent, democratic and reproducible process and is recognised as the gold standard in setting research priorities. The JLA is a UK-based non-profit initiative created in 2004 by the National Institute of Health Research Evaluation, Trials and Studies Coordinating Centre. ${ }^{11}$ The JLA PSP process requires active participation from patients, patient-support organisations and clinicians, on an equal basis, in order to highlight unanswered questions regarding therapeutic options in a specific disease state. Over 30 disease areas have been subjected to a JLA PSP process, such as diabetes and dementia. ${ }^{12} 13$ The JLA-based PSP should include at least the following five stages. A steering group is composed of key representatives from relevant healthcare organisations and from patient and patientsupport groups (stage 1). The scope and timeline of the PSP are then established (stage 2), followed by the collection of potential research questions through anonymous surveys (stage 3 ). The treatment uncertainties are then collated and summarised by members of the steering group (stage 4). The 'top 10' list of
Box 1 Top 10 research priorities in inflammatory bowel disease (IBD) resulting from the James Lind Alliance Priority Setting Partnership in 2016

1. What is the optimal treatment strategy considering efficacy, safety and cost-effectiveness (immunomodulators, biologics, surgery, combinations) in IBD management: selecting the right patient group, right stage of disease, and assessing potential for withdrawal?

2. What are the optimal markers/ combinations of markers (clinical, endoscopic, imaging, genetics, other biomarkers) for stratification of patients with regards to $(A)$ disease course and (B) monitoring disease activity and (C) treatment response?

3. What role does diet have in the management of mildly active or inactive ulcerative colitis or Crohn's disease to achieve normal daily activities and symptom control?

4. How can pain be most effectively managed in people with IBD?

5. What is an optimal treatment strategy for perianal Crohn's disease and what individual factors determine this?

6. What is the best treatment for controlling diarrhoea and/or incontinence symptoms in people with IBD, including novel pharmacological and non-pharmacological options? Is high-dose loperamide safe and effective in the treatment of diarrhoea in IBD?

7. What is the optimal dietary therapy (liquid enteral diet and/or reintroduction diet) and duration to achieve mucosal healing in active IBD and/or remission either as a primary or adjunctive treatment? Is there a difference between adults and children?

8. What is the association between IBD and fatigue and how should it be managed?

9. Does early surgery or later surgery for terminal ileal Crohn's disease result in better outcomes (quality of life, cost-effectiveness)?

10. Does influencing the gut microbiota influence the course of IBD?

research priorities are then determined by the JLA participants through face-to-face workshops designed to reach general consensus in a stepwise fashion (stage 5). ${ }^{14}$ Non-clinical academic researchers and the pharmaceutic industry are not involved in any of the five stages to ensure that the identified research priorities were the ones that are most important to the patients and their healthcare professionals. ${ }^{2}{ }^{14}$

The steering committee of the UK IBD collaborative partnership included two IBD patients, two gastroenterologists, two IBD specialist nurses, two colorectal surgeons, two dieticians, a representative from the UK IBD charity organisation Crohn's and Colitis UK, a representative of the JLA and an administrator. This initiative was the first in its kind in gastroenterology and the final output was the publication of 10 key research priorities, ranked in order of perceived importance (see box 1). ${ }^{3}$

Six years after initiation of the JLA PSP process for IBD therapies and over 3 years after the publication 
of the top 10 IBD-research priorities, the question remains whether this initiative has had an impact on the research landscape. Therefore, the aim of this study is to create an overview of the current distribution over these top 10 research domains of all clinical trials in adults with IBD ongoing or completed in the UK within the past 3 years.

\section{METHODS}

The database from ClinicalTrials.gov and European Union Clinical Trials Register was used to create a list of all clinical studies set up in the UK involving adult patients with IBD. Trials posted on 9 August 2016 (publication date of the top 10 research questions) up to 16 November 2019 were included. Any relevant studies known to the authors and not found in this search were added to the list. Pharmaceutical industry-sponsored trials were recorded in a separate list given their inherent bias towards drug conception and approval. For each trial, data were collected on study type, sponsor and progression status. If the study was finished, then the PubMed database was searched for a corresponding publication. All study data were exported as an SPSS statistics 26 file for analysis.

\section{RESULTS}

In total, 20 non-pharmaceutical-funded clinical studies in the UK were found (online supplemental table 1) of which eight were randomised clinical trials, six were non-randomised (open-label) clinical trials and six were observational studies. All studies addressed at least one of the top 10 IBD-research priorities. The IBD-BOOST trial, the PREdiCCt study and the MODULATE trial addressed multiple priorities simultaneously.
The distribution of these studies per research priority number is given in figure 1. A quarter of the nonpharmaceutical-driven IBD studies investigated the optimal treatment strategy considering efficacy, safety and cost-effectiveness (immunomodulators, biologics, surgery) in IBD (priority 1). Four studies evaluated the role of diet in IBD management (priorities 3 and 7). Development or assessment of biomarkers for stratification of patients (priority 2) was subject of three studies, although no study focused on development of markers for treatment response (priority $2 \mathrm{c}$ ).

Important patient-reported outcomes such as IBDrelated pain and control of diarrhoea and incontinence were each subject of two studies (respectively, priorities 4 and 6). Only one study (MODULATE trial) evaluated the effectiveness of loperamide in IBD (part of priority 6). Three studies focused on the association between fatigue and IBD and its management (priority 8).

Measuring the effect of altered gut microbiota on the evolution of IBD (priority 10) and determining the optimal treatment strategy for perianal Crohn's disease (priority 5) was the target of two studies each. One study assessed surgical treatment for terminal ileal Crohn's disease (priority 9).

Nineteen out of 20 studies were initiated by investigators from the UK and exclusively carried out in the UK. Only the LIR!C trial was led by a sponsor outside the UK and with participating UK sites. Of all studies, two were finished at the time of data collection, of which only the LIR!C trial was published, which was led by Dutch researchers with contribution from UK-based investigators. ${ }^{15}$ The other completed study was a feasibility study prior to the CD-TREAT trial and was not published. The STOP-Colitis pilot trial

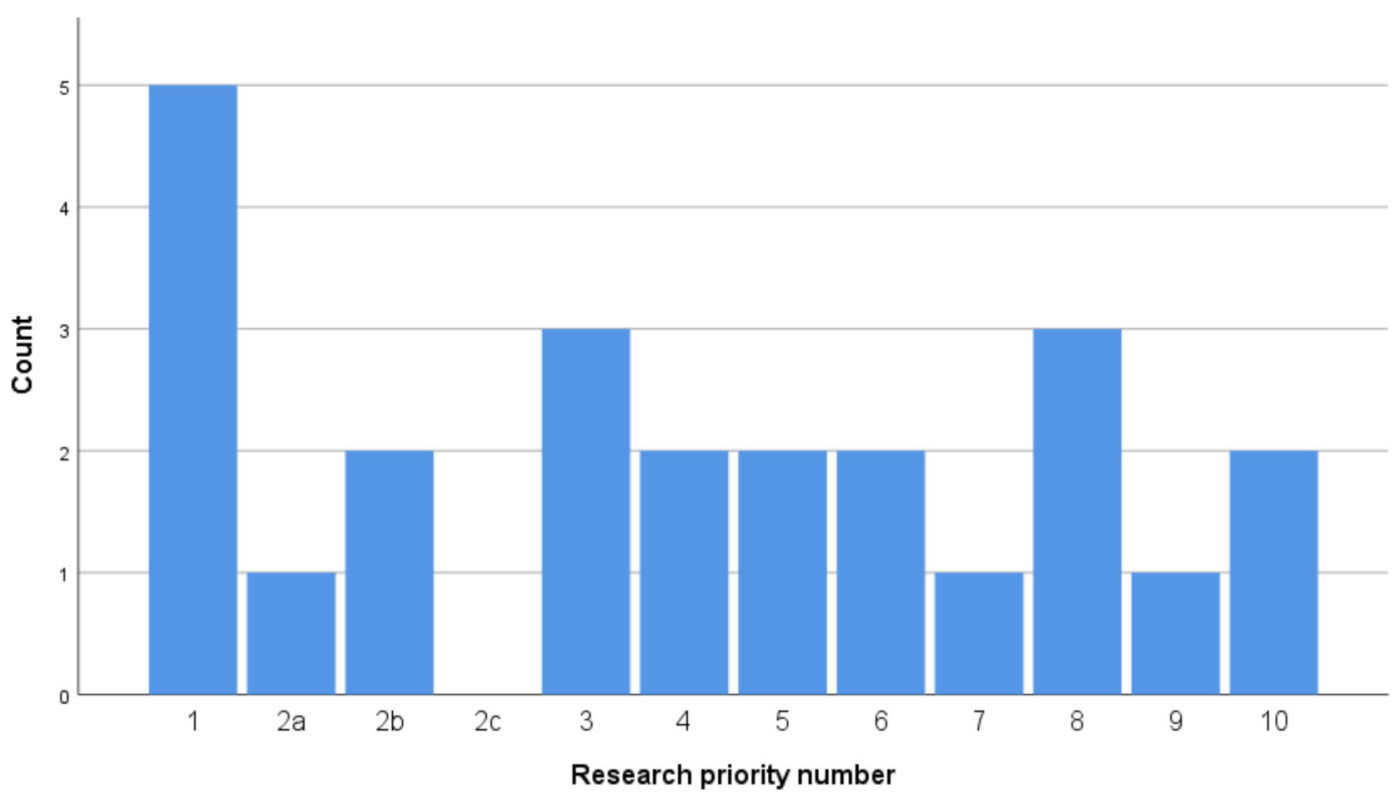

Figure 1 Number of non-pharmaceutical sponsored studies on adult patients per JLA-IBD research priority since 9 August 2016 in the UK $(n=20-3$ studies focused on more than one priority). JLA-IBD, James Lind Alliance-inflammatory bowel disease. 


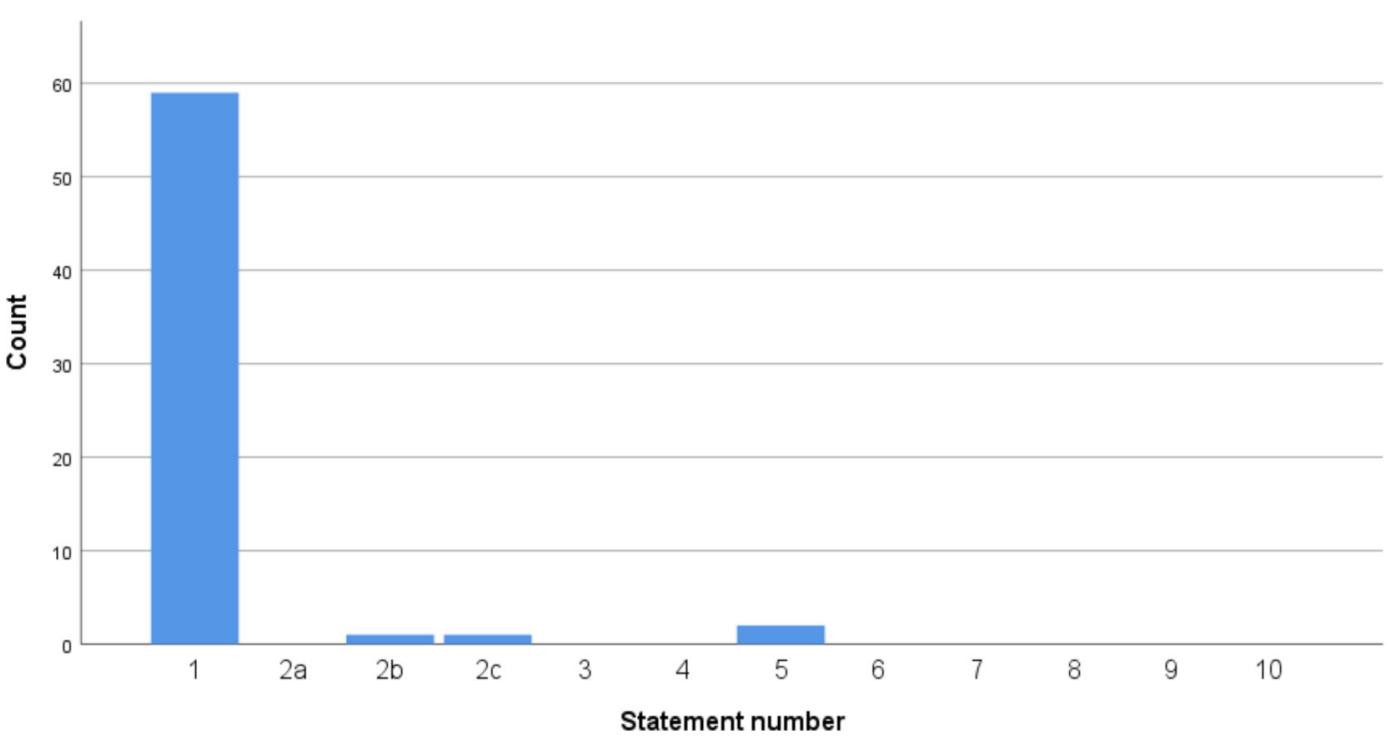

Figure 2 Number of pharmaceutical industry sponsored studies on adult patients per JLA-IBD research priority since 9 August 2016 in the UK $(n=63)$. JLA-IBD, James Lind Alliance-inflammatory bowel disease.

protocol was published but the study itself is ongoing at the time of enquiry. ${ }^{16}$ Meanwhile, in December 2019, the pilot trial on cognitive-behavioural therapy for the management of IBD-fatigue by Artom et al has also been published. ${ }^{17}$

The same evaluation was done for pharmaceuticaldriven studies in the past 3 years. In total, 63 studies were found using the ClinicalTrials.gov database relating to at least one of the JLA Top 10 research priorities. The vast majority of these studies $(59 / 63)$ focused on priority 1 concerning development and validation of new medical treatments (see figure 2). Of all 63 studies, six were finished at the moment of enquiry (online supplemental table 2).

\section{DISCUSSION}

The results of this study confirm that, as to be expected, industry-sponsored research is mainly focused on development and validation of new medical therapies (priority 1). Other research priorities, in particular addressing symptom management which is important to patients, are mainly overlooked by the industrydriven research. As for the non-industry-sponsored studies, the distribution follows the order of the priority ranking. Priority number 1 remains the most frequently targeted study subject, accounting for $25 \%$ of all studies. The role of dietary interventions in IBD is gaining interest and was subject of $20 \%$ of all studies. Some non-industry-sponsored studies focused on patient-reported outcomes such as IBD-related pain (two studies), fatigue (three studies) and diarrhoea/ incontinence (two studies), but these studies accounted for only a small proportion of all the trials. As a consequence, it remains debatable to what extent the current landscape of clinical trials adequately represents the patients' viewpoint on needs for expanded knowledge in IBD and thus, it might be suggested that these research subjects should be prioritised in the near future.

This study has some important limitations and therefore it is difficult to draw robust conclusions from this overview. First of all, the search for trials was restricted to the UK since development of the JLA-IBD Top 10 research priorities was a solely UK initiative. Second, the number of retrieved studies not sponsored by industry is small. Furthermore, the search was limited to the ClinicalTrials.gov database and the European Union Clinical Trials Register. Any relevant studies known to the authors were added to the list. Given the possible report bias, we acknowledge that this overview of studies might be incomplete. Last but not least, the time frame of evaluation is relatively short and the question remains to what extent the message of the publication of the 10 IBD research priorities had been spread throughout the research landscape before the setup of the investigated studies, especially in the first year after publication. Therefore, no assumptions can be made on any sort of causality between the publication of the top 10 priorities and the current distribution of studies over the different priority subjects.

Nonetheless, the development of the top 10 priorities using the JLA framework should be seen as a milestone in streamlining IBD research towards better, patient-centred care. The potential benefit of this PSP has gained interest outside the UK, which is illustrated by a similar initiative in Canada resulting in development of a list of priorities in paediatric IBD research. ${ }^{18}$

The role of patient and public involvement (PPI) in research has expanded over the last twenty years and has been shown to improve study recruitment and participation, as well as clinical relevance. ${ }^{19-21} \mathrm{In}$ the UK, a majority of grant applications must include a documented plan for PPI, however, this does not necessarily translate to increased PPI reporting within 
manuscript submission to medical journals. Indeed, the $B M J$ reported PPI activity in $0.5 \%$ of submitted research papers from 1 June 2013 to 31 May 2014, which then increased to $11 \%$ from 1 June 2015 to 31 May 2016 after the introduction of a mandatory policy to include a PPI declaration in the Methods section. ${ }^{22}$ Addressing the research priorities most important to patients and clinicians can be achieved by initiatives such the BMJ's and other medical journals, in collaboration with funding bodies, research institutions and charitable organisations such as the Crohn's and colitis UK.

This qualitative review also highlights that many relevant research questions remain unanswered. For instance, further research could investigate how the impact of these PSPs can be evaluated in a standardised way, as well as determine the time frame for revision of the priority ranking. There may be value in creating a centralised registry of all studies classified by priority topic. This study may provide evidence where there remains a relative lack of funding in IBD. This could help raise stakeholder awareness of where investments need to be made in order to address the unmet needs in IBD care.

\section{CONCLUSION}

This study aimed to assess the impact of the 2016 PSP between clinicians, patients and patient-support organisations using the JLA framework for IBD research in the UK. For this purpose, an overview of all clinical studies concerning at least one of the top 10 research priorities in IBD was created. All relevant clinical studies set up and ongoing in the UK since publication of these top 10 research priorities in August 2016 were considered. Of the 63 retrieved industry-sponsored studies, the vast majority focused on development and validation of new medical therapies (priority 1 ). The distribution of non-industry-sponsored study targets is more spread over the 10 research priorities. However, very few studies focus on patient-reported outcomes. As a consequence, and despite clear progress across multiple domains, it remains debatable to what extent the current landscape of clinical trials in the UK adequately represents the viewpoint of all stakeholders, especially patients.

Contributors JG: conducted data collection, wrote and reviewed manuscript, submitted manuscript, J-FL: conducted data collection, wrote and reviewed manuscript. J-FL: conducted data collection, wrote and reviewed manuscript. JS: wrote and reviewed manuscript. CWL: developed study, reviewed manuscript, responsible for the overall content. $\mathrm{AH}$ : developed study, reviewed manuscript, responsible for the overall content. Both authors contributed equally as first authors. Both authors contributed equally as last authors

Funding The authors have not declared a specific grant for this research from any funding agency in the public, commercial or not-for-profit sectors.

Competing interests JG, J-FL, LL: no competing interests. JS: was actively involved in the trial 'STOP-Colitis pilot trial protocol: a prospective, open-label, randomised pilot study to assess two possible routes of faecal microbiota transplant delivery in patients with ulcerative colitis. 'CWL: is actively involved in the following trial: 'The PRognostic Effect of Environmental Factors in Crohn's and Colitis (PREdiCCt)'AL Hart: is actively involved in the following trials: • 'STOP-Colitis pilot trial protocol: a prospective, open-label, randomised pilot study to assess two possible routes of faecal microbiota transplant delivery in patients with ulcerative colitis' • 'A supported online self-management tool for symptoms of fatigue, pain and urgency/incontinence in people with inflammatory bowel disease: the IBD-BOOST trial'.

\section{Patient consent for publication Not required.}

Provenance and peer review Not commissioned; externally peer reviewed.

Data availability statement Data are available in a public, open access repository. Data are available on reasonable request. All data relevant to the study are included in the article or uploaded as online supplemental information. Data were extracted from ClinicalTrials.gov and European Union Clinical Trials Register. Please see the 'Methods' from the main manuscript.

Supplemental material This content has been supplied by the author(s). It has not been vetted by BMJ Publishing Group Limited (BMJ) and may not have been peer-reviewed. Any opinions or recommendations discussed are solely those of the author(s) and are not endorsed by BMJ. BMJ disclaims all liability and responsibility arising from any reliance placed on the content. Where the content includes any translated material, BMJ does not warrant the accuracy and reliability of the translations (including but not limited to local regulations, clinical guidelines, terminology, drug names and drug dosages), and is not responsible for any error and/or omissions arising from translation and adaptation or otherwise.

\section{ORCID iDs}

Jeroen Geldof http://orcid.org/0000-0003-0126-2929

Jonathan Segal http://orcid.org/0000-0002-9668-0316

\section{REFERENCES}

1 Dalziel TK. Chronic interstitial enteritis. $\mathrm{Br} \mathrm{Med} J$ 1913;2:1068-70.

2 Mulder DJ, Noble AJ, Justinich CJ, et al. A tale of two diseases: the history of inflammatory bowel disease. J Crohns Colitis 2014;8:341-8.

3 Hart AL, Lomer M, Verjee A, et al. What are the top 10 research questions in the treatment of inflammatory bowel disease? A priority setting partnership with the James Lind alliance. J Crohns Colitis 2017;11:204-11.

4 Khor B, Gardet A, Xavier RJ. Genetics and pathogenesis of inflammatory bowel disease. Nature 2011;474:307-17.

5 SVARTZ N. Salazopyrin, a new sulfanilamide preparation. A. therapeutic results in rheumatic polyarthritis. B. therapeutic results in ulcerative colitis. C. toxic manifestations in treatment with sulfanilamide preparations. Acta Med Scand 1942;110:577-98.

6 TRUELOVE SC, WITTS LJ. Cortisone in ulcerative colitis; final report on a therapeutic trial. Br Med J 1955;2:1041-8.

7 BEAN RH. The treatment of chronic ulcerative colitis with 6-mercaptopurine. Med J Aust 1962;49:592-3.

8 Rutgeerts P, Sandborn WJ, Feagan BG, et al. Infliximab for induction and maintenance therapy for ulcerative colitis. $\mathrm{N}$ Engl J Med 2005;353:2462-76.

9 Kurti Z, Vegh Z, Golovics PA, et al. 'Treat to Target' - Lessons Learnt. Dig Dis 2016;34:147-52.

10 Morar P, Read J, Arora S, et al. Defining the optimal design of the inflammatory bowel disease multidisciplinary team: results from a multicentre qualitative expert-based study. Frontline Gastroenterol 2015;6:290-7.

11 Alatab, S, GBD 2017 Inflammatory Bowel Disease Collaborators. The global, regional, and national burden of inflammatory bowel disease in 195 countries and territories, 
1990-2017: a systematic analysis for the global burden of disease study 2017. Lancet Gastroenterol Hepatol 2020;5:1730.

12 de Souza HSP, Fiocchi C, Iliopoulos D. The IBD interactome: an integrated view of aetiology, pathogenesis and therapy. Nat Rev Gastroenterol Hepatol 2017;14:739-49.

13 Peyrin-Biroulet L, Sandborn W, Sands BE, et al. Selecting therapeutic targets in inflammatory bowel disease (STRIDE): determining therapeutic goals for Treat-to-Target. Am J Gastroenterol 2015;110:1324-38.

14 Higgins PDR. The development of patient-reported outcome measures in inflammatory bowel disease. Gastroenterol Hepatol 2018;14:658.

15 de Groof EJ, Stevens TW, Eshuis EJ, et al. Cost-effectiveness of laparoscopic ileocaecal resection versus infliximab treatment of terminal ileitis in Crohn's disease: the LIR!C Trial. Gut 2019;68:1774-80.

16 Quraishi MNN, Yalchin M, Blackwell C, et al. STOP-Colitis pilot trial protocol: a prospective, open-label, randomised pilot study to assess two possible routes of faecal microbiota transplant delivery in patients with ulcerative colitis. BMJ Open 2019;9:e030659.
17 Artom M, Czuber-Dochan W, Sturt J, et al. CognitiveBehavioural therapy for the management of inflammatory bowel disease-fatigue: a feasibility randomised controlled trial. Pilot Feasibility Stud 2019;5:145.

18 Grant A, Crane M, Laupacis A, et al. Engaging patients and caregivers in research for pediatric inflammatory bowel disease: top 10 research priorities. J Pediatr Gastroenterol Nutr 2019. ;;69:317-23. 2019 Sep.

19 Bailey S, Boddy K, Briscoe S, et al. Involving disabled children and young people as partners in research: a systematic review. Child Care Health Dev 2015;41:505-14.

20 Fudge N, Wolfe CDA, McKevitt C. Involving older people in health research. Age Ageing 2007;36:492-500.

21 Hanney S, Boaz A, Jones T, et al. Engagement in research: an innovative three-stage review of the benefits for health-care performance. Health Services and Delivery Research 2013;1:1152.

22 Price A, Schroter S, Snow R, et al. Frequency of reporting on patient and public involvement $(\mathrm{PPi})$ in research studies published in a general medical Journal: a descriptive study. BMJ Open 2018;8:e020452. 